\title{
Catamenial pneumothorax: multidisciplinary minimally invasive management of a recurrent case
}

\author{
Cihan Kaya ${ }^{1}$, Derya Ece Iliman $^{1}$, Gun Murat Eyuboglu², Ece Bahceci ${ }^{1}$ \\ ${ }^{1}$ Obstetrics and Gynecology Department, Bakirkoy Dr. Sadi Konuk Research and Training Hospital, Istanbul, Turkey \\ ${ }^{2}$ Thoracic Surgery Department, Bakirkoy Dr.Sadi Konuk Research and Training Hospital, Istanbul, Turkey
}

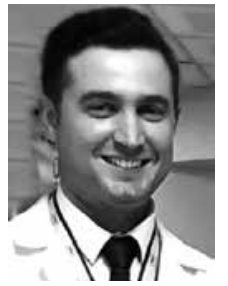

Kardiochirur Torakochir Pol 2020; 17 (2): 107-109

Thoracic endometriosis syndrome (TES) is the presence of endometriotic foci within the respiratory system and involves a range of symptoms that coincide with the menses. These symptoms are defined as the presence of pneumothorax, hemothorax, hemoptysis, lung nodules, isolated chest pain, and pneumomediastinum. Catamenial pneumothorax (CP) is described as recurrent abnormal air collection between the lung and chest wall that occurs within the first 72 hours of the menses and is the most common presentation of TES [1].

Although it is considered quite a rare condition, 490 patients have been reported so far. According to the current evidence, its prevalence is as high as $30 \%$ in women with spontaneous pneumothorax [2, 3]. Due to the lack of data in the literature, there is no definite information about the management of recurrent cases.

In this report, we present a case with recurrent $\mathrm{CP}$ resistant to a variety of medical treatments managed via a minimally invasive approach by a multidisciplinary team.

A 36-year-old, primiparous woman presented with persistent right shoulder pain and cyclic hemoptysis. She had a history of seven $C P$ episodes requiring pleurodesis in three of them. Her symptoms persisted, despite having 6 months of cyclic OC treatment, dienogest for 6 months, and two courses of depot GnRHa. Her pelvic examination was unremarkable, and a $3 \mathrm{~cm}$ endometrioma was observed on her transvaginal ultrasound (TVUSG) examination. A right pleural thickening was observed in computed tomography (CT) (Figure 1). After consulting anesthesiology and thoracic surgery departments, an operative laparoscopy for peritoneal/diaphragmatic endometriosis and video-assisted thoracoscopy (VATS) was planned to treat recurrent $\mathrm{CP}$ episodes.

A double-lumen tube was used by an anesthesiologist to create a controlled collapse of the right lung for exploring the chest cavity. After achieving adequate pneumoperitoneum, a 10-mm intra-umbilical optical trocar was inserted. Two lateral and one suprapubic 5-mm accessory trocars were then placed. An ultrasonic scalpel was used in addition to standard laparoscopic equipment.
On surgical exploration, about 2-3 cm endometriotic nodules were observed on paravesical leaves of the broad ligament. Ureterolysis was performed before the excision of endometriotic nodules located on both of the sacrouterine ligaments. A $3 \mathrm{~cm}$ endometrioma was observed on the right ovary, and right salpingo-oophorectomy was performed.

Multiple superficial endometriotic lesions were observed on the diaphragmatic peritoneal surface. Considering the patient's complaints, we resected a wide peritoneal surface of the diaphragm after blunt dissection by a monopolar hook.

The VATS procedure was performed to identify any lung involvement and pleural involvement by a thoracic surgeon. On exploration, a $6 \times 5 \mathrm{~cm}$ pleural nodule surrounded with multiple small endometriotic lesions was observed. The borders of the nodule were marked with a monopolar hook, and it was removed with a curved surgical clamp (Figure 2).

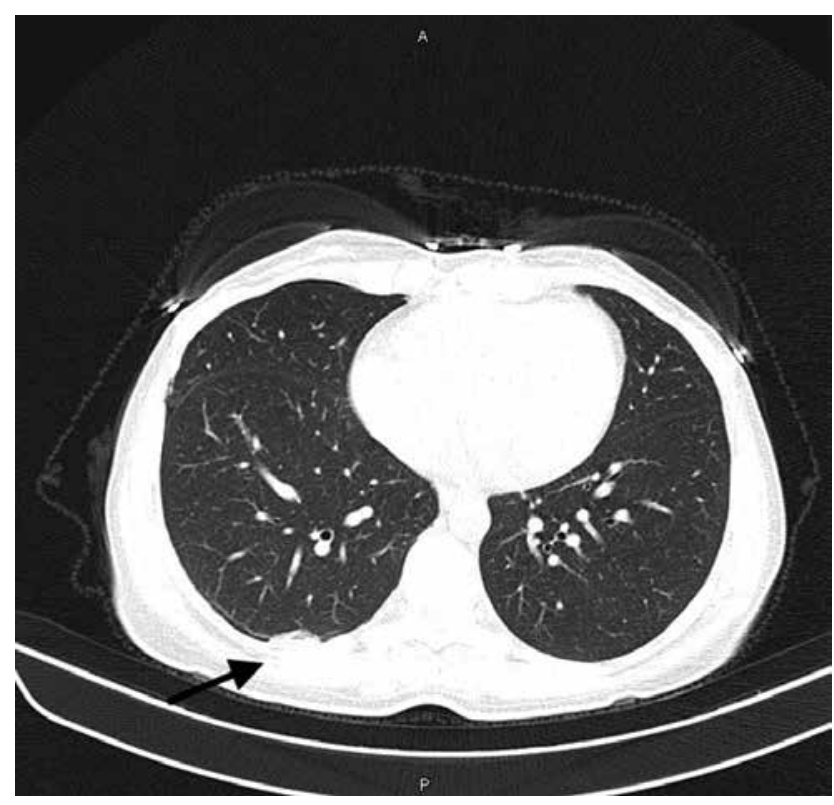

Figure 1. Computed tomography image. The black arrow shows pleural thickening on the right side

Address for corresepondence: Derya Ece Iliman MD, Obstetrics and Gynecology Department, Bakirkoy Dr. Sadi Konuk Research and Training Hospital, Istanbul, Turkey, phone: +90 5066698815, e-mail: deryaeceiliman@gmail.com

Received: 20.05.2020, accepted: 10.06.2020. 

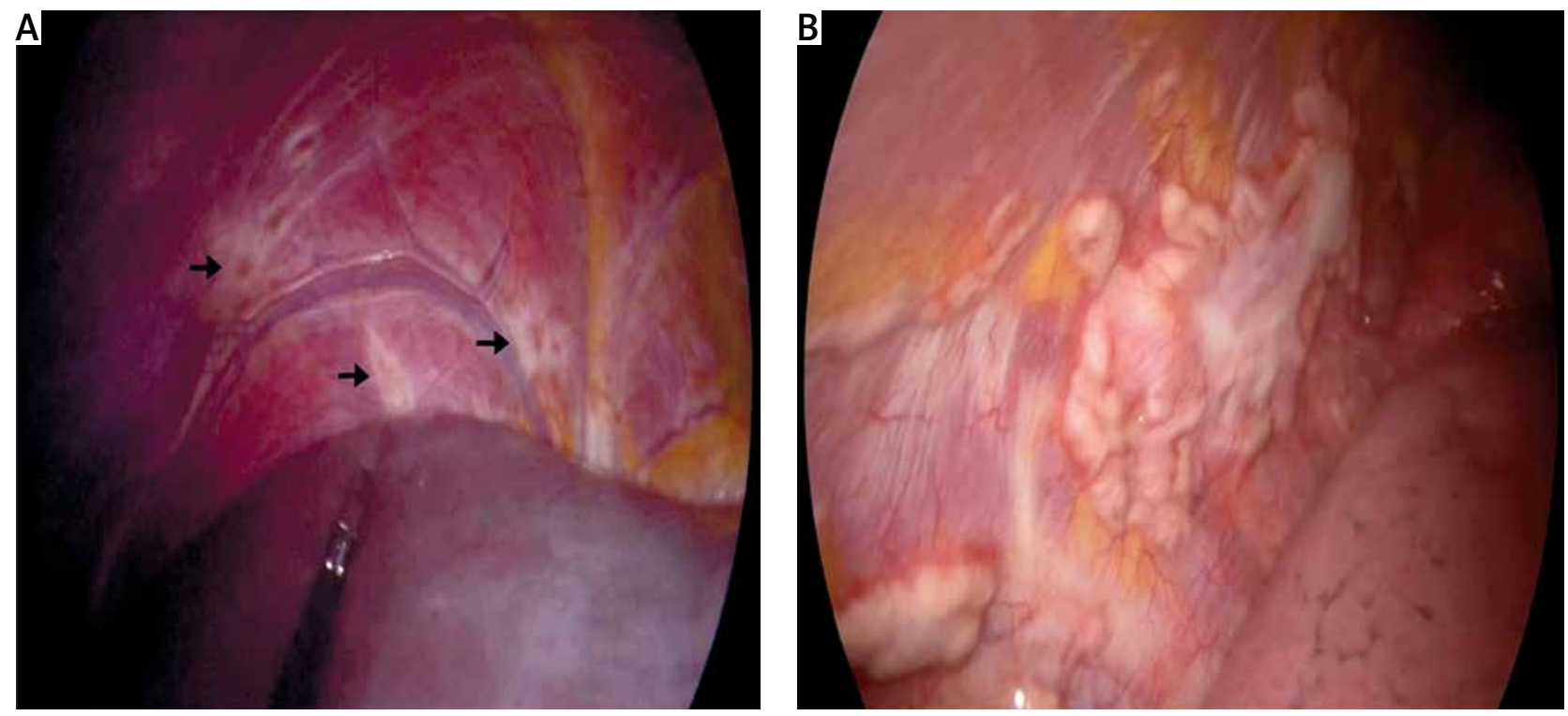

Figure 2. A - Laparoscopic image. Black arrows show multiple superficial endometriotic lesions on the diaphragmatic peritoneal surface. B - VATS view. A $6 \times 5 \mathrm{~cm}$ pleural nodule

A wedge resection was performed with a stapler due to a $0.5 \mathrm{~cm}$ suspicious endometriotic lesion placed on the lower right lung segment. After achieving hemostasis, collagen barriers were placed to have better tissue healing. A $32 \mathrm{f}$ thoracostomy tube was placed for drainage. The postoperative course was uneventful. A 6-month depot $\mathrm{GnRH}$ analogue treatment was planned to reduce postoperative recurrence.

Although various theories have been proposed to explain the pathogenesis of TE [4], up to now, none can explain all features of the disease. These theories include coelomic metaplasia, retrograde menstruation, migration of endometrial tissue, stem cell theory, microembolization through the pelvic veins, and abnormal lymphatic drainage. It is commonly believed that the underlying cause is multifactorial.

CP is known as a typical symptom of TES, but it can occur with no visible signs of thoracic endometriosis, and CP is the primary symptom of these patients [1-5]. Current literature mostly consists of case reports and small retrospective studies. Ninety-five percent of cases occur at the right hemithorax, but left-sided and bilateral involvement also has been described. The recurrence rate of CP was found to be $30-39 \%$ in various studies [5]. TES was found to be associated with pelvic endometriosis (PE), and hemothorax was the most frequent symptom of these patients [1-5]. $20-70 \%$ of CP patients have been reported to experience pelvic endometriosis [3-5]. As seen in our case, difficulties in the diagnosis, absence of a multidisciplinary team, and delay in treatment could reduce the patients' quality of life.

Currently, no guidelines have been released regarding the appropriate diagnosis and treatment of TE. Acute CP treatment is essentially surgical. The VATS procedure is mostly recommended to visualize any endometriotic lesions on the pleura and any defects on the diaphragm [3].
The current evidence suggests that resection or electrocoagulation of endometriotic lesions may help to prevent further spreading and disease control. Other recommended surgical techniques include wedge resection, removal of blebs, diaphragmatic resection, and repair via patch, mesh, or simple suturing. One prospective study, including 32 patients, suggested that diaphragmatic defects can play a crucial role in recurrent CP cases, and these defects almost always occur on the right side of the diaphragm [6].

The recurrence rates of CP after surgical treatment are relatively high. In addition to surgery, suppressive medical treatment can also be used in the postoperative period. For that purpose, various hormonal therapies such as cyclic estrogen-progesterone combinations, GnRH agonists, danazol, and progesterone-only drugs were used. The most effective agent was reported to be a GnRH agonist, but due to the menopausal side effects of this treatment, it was only recommended for short-term use in the pre- or postoperative period [3]. Combined oral contraceptive (COC) treatments are mostly found ineffective, as in our patient. Yet, there is no reliable evidence on the optimal suppressor agent, dose regimen, or the success rates [3].

Recurrence may also occur after surgical treatment. Talc pleurodesis was found highly effective, yet using this treatment with non-malignant patients is still controversial [7]. In a recent study, it was reported that a high rate of recurrence was observed after treatment with abrasion pleurodesis alone. In our patient, despite having three courses of pleurodesis treatment, recurrence was observed. We may suggest that to reduce recurrence rates, removal of all visible endometriotic tissue is more feasible than pleurodesis alone. Bagan et al. indicated that diaphragmatic repair could lower the rate of recurrence and improves outcomes. They also recommended that covering the surface of the diaphragm with a polyglactin mesh was recom- 
mended, even if there are no visible diaphragmatic lesions or defects to reduce recurrence rates [4].

To sum up, CP is a diagnosis that can be easily overlooked. Patients presenting with pneumothorax at reproductive age should always be considered with this in mind. Treatment options can differ according to patient history, desire to conceive, and persistence of episodes or symptoms. The medical and surgical combination treatments should be considered in the foreground when appropriate. The management of a multidisciplinary team may give these patients a chance of a minimally invasive approach.

\section{Disclosure}

Authors report no conflict of interest.

\section{References}

1. Joseph J, Sahn SA. Thoracic endometriosis syndrome: new observations from an analysis of 110 cases. Am J Med 1996; 100: 164-170.

2. Haga T, Kataoka $\mathrm{H}$, Ebana $\mathrm{H}$, et al. Thoracic endometriosis-related pneumothorax distinguished from primary spontaneous pneumothorax in females. Lung 2014; 192: 583-587.

3. Gil Y, Tulandi T. Diagnosis and treatment of catamenial pneumothorax: a systematic review. J Minim Invasive Gynecol 2020; 27: 48-53.

4. Bagan P, Le Pimpec Barthes F, Assouad J, et al. Catamenial pneumothorax retrospective study of surgical treatment. Ann Thorac Surg 2003; 75: 378-381.

5. Haga T, Kurihara M, Kataoka H, Ebana $H$. Clinical-pathological findings of catamenial pneumothorax: comparison between recurrent cases and nonrecurrent cases. Ann Thorac Cardiovasc Surg 2014; 20: 202-206.

6. Alifano M, Roth T, Broet SC, et al. Catamenial Pneumothorax. Chest 2003; 124: 1004-1008.

7. Alifano M, Trisolini R, Cancellieri A, et al. Thoracic endometriosis: current knowledge. Ann Thorac Surg 2006; 81: 761-769. 